\title{
Mathematical Studies of Dynamics and Evolution of Infectious Diseases
}

Yoh Iwasa, Kazunori Sato, and Yasuhiro Takeuchi

The practical importance of understanding the dynamics and evolution of infectious diseases is steadily increasing in the contemporary world. One of the most important mortality factors for the human population is malaria. Every year, hundreds of millions of people suffer from malaria, and more than a million children die. One of the obstacles of controlling malaria is the emergence of drug-resistant strains. Pathogen strains resistant to antibiotics pose an important threat in developing countries. In addition, we observe new infectious diseases, such as HIV, Ebora, and SARS.

The mathematical study of infectious disease dynamics has a long history. The classic work by Kermack and McKendrick (1927) established the basis of modeling infectious disease dynamics. The variables indicate the numbers of host individuals in several different states - susceptive, infective and removed. This formalism is the basis of all current modeling of the dynamics and evolution of infectious diseases. Since then, the number of theoretical papers on infectious diseases has increased steadily. Especially influential was a series of papers by Roy Anderson and Robert May, summarized in their book (Anderson and May 1991). Anderson and May have developed population dynamic models of the host engaged in reproduction and migration. In a sense, they treated epidemic dynamics as a variant of ecological population dynamics of multiple species community. Combining the increase of our knowledge of nonlinear dynamical systems (e.g. chaos), Anderson and May also demonstrated the usefulness of simple models in understanding the basic principles of the system, and sometimes even in choosing a proper policy of infectious disease control.

The dynamical systems for epidemics are characterized by nonlinearity. The systems include many processes at very different scales, from the population on earth to the individual level, and further to the immune system within a patient. Hence, mathematical studies of epidemics need to face this dynamical diversity of phenomena. Tools of modeling and analysis for situations including time delay and spatial heterogeneity are very important. As a consequence, there is no universal mathematical model that holds for all 
problems in epidemics. When we are given a set of epidemiological phenomena and questions to answer, we must "construct" mathematical models that can describe the phenomena and answer our questions. This is quite different from studies in "pure" mathematics, in which usually the models are given beforehand.

One of the most important questions in mathematical studies of epidemics is the possibility of the eradication of disease. The standard local stability analysis of the endemic equilibrium and disease-free equilibrium is often not enough to answer the question, because it gives us information only on the local behavior, or the solution in the neighborhood of those equilibria. On the other hand, it is known that global stability analysis of the models is often very difficult, and even impossible in general cases, because the dynamics are highly nonlinear. Even if the endemic equilibrium were unstable and the disease-free equilibrium were locally stable, the diseases can remain endemic and be sustained forever. Sometimes, rather simple models show periodic or chaotic behavior. Recently, the concept of "permanence" was introduced in population biology and has been studied extensively. This concept is very important in mathematical epidemiology as well. Permanence implies that the disease will be maintained globally, irrespective of the initial composition. Even if the endemic equilibrium were unstable, the disease will last forever, possibly with perpetual oscillation or chaotic fluctuation.

Since the epidemiological data supplied by medical and public health sectors are abundant, epidemiological models are in general much better tested than similar population models in ecology developed for wild animals and plants. The diversity of models is also extensive, including all the different levels of complexity. Rather simple and abstract models are suitable to discuss general properties of the system, while more complex and realistic computerbased simulators are adopted for policy decision making incorporating details of the structure closely corresponding to available data. Mathematical modeling of infectious diseases is the most advanced subfield of theoretical studies in biology and the life sciences. What is notable in this development is that, even if many computer-based detailed simulators become available, the rigorous mathematical analysis of simple models remains very useful, medically and biologically, in giving a clear understanding of the behavior of the system.

Recently, the evolutionary change of infectious agents in the host population or within a patient has attracted an increasing attention. Mutations during genome replication would create pathogens that may differ slightly from the original types. This gives an opportunity for a novel strain to emerge and spread. As noted before, emergence of resistant strains is a major obstacle of infectious disease control. Essentially the same evolutionary process occurs within the body of a single patient. A famous example is HIV, in which viral particles change and diversify their nucleotide sequences after they infect a patient. This supposedly reflects the selection by the immune system of the host working on the virus genome. A similar process of escape is involved 
in carcinogenesis - a process in which normal stem cells of the host become cancerous.

The papers included in this volume are for mathematical studies of models on infectious diseases and cancer. Most of them are based on presentations in the First International Symposium on Dynamical Systems Theory and its Applications to Biology and Environmental Sciences, held in Hamamatsu, Japan, on 14-17 March 2004. This introductory chapter is followed by four papers on infectious disease dynamics, in which the roles of time delay (Chaps. 2 and 3) and spatial structures (Chaps. 4 and 5) are explored. Then, there are two chapters that discuss competition between strains and evolution occurring in the host population (Chap. 6) and within a single patient (Chap. 7). Finally, there are papers on models of the immune system and cancer (Chaps. 8 and 9). Below, we briefly summarize the contents of each chapter.

In Chap. 2, Zhien Ma and Jianquan Li give an introduction to the mathematical modeling of disease dynamics. Then, they summarize a project of modeling the spread of SARS in China by the authors and their colleagues.

In Chap. 3, Yasuhiro Takeuchi and Wanbiao Ma introduce mathematical studies of models with time delay. They first review past mathematical studies on this theme during the last few decades, and then introduce their own work on the stability of the equilibrium and the permanence of epidemiological dynamics.

In Chaps 4 and 5, Wendi Wang and Shigui Ruan discuss the spatial aspect of epidemiology. The spread of a disease in a population previously not infected may appear as "wave of advance". This is often modeled as a reaction diffusion system, or by other models handling spatial aspects of population dynamics. The speed of disease propagation is analogous to the spread of invaders in a novel habitat in spatial ecology (Shigesada and Kawasaki 1997).

Since microbes have a shorter generation time and huge numbers of individuals, they have much faster evolutionary changes, causing drug resistance and immune escape, among the most common problems in epidemiology. By considering the appearance of novel strains with different properties from those of the resident population of pathogens, and tracing their abundance, we can discuss the evolutionary dynamics of infectious diseases. In Chap. 6, Horst Thieme summarized the work on the competition between different and competing strains, and the possibility of their coexistence and replacement. An important concept is the "maximal basic replacement ratio". If a host once infected and then recovered from a single strain is perfectly immune to all the other strains (i. e. cross immunity is perfect), then the one with the largest basic replacement ratio will win the competition among the strains. The author explores the extent to which this result can be generalized. He also discusses the coexistence of strains considering the aspect of maternal transmission as well.

In Chap. 7, Yoh Iwasa and his colleagues analyze the result of evolutionary change occurring within the body of a single patient. Some of the 
pathogens, especially RNA viruses have high mutation rates, due to an unreliable replication mechanism, and hence show rapid genetic change in a host. The nucleotide sequences just after infection by HIV will be quite different from those HIV occurring after several years. By mutation and natural selection under the control of the immune system, they become diversified and constantly evolve. Iwasa and his colleagues derive a result that, without cross-immunity among strains, the pathogenicity of the disease tends to increase by any evolutionary changes. They explore several different forms of cross-immunity for which the result still seems to hold.

In Chap. 8, Edoardo Beretta and his colleagues discuss immune response based on mathematical models including time delay. The immune system has evolved to cope with infectious diseases and cancers. They have properties of immune memory and, once attached and recovered, they will no longer be susceptive to infection by the same strain. To achieve this, the body has a complicated network of diverse immune cells. Beretta and his colleagues summarize their study of modeling of an immune system dynamics in which time delay is incorporated.

In the last chapter, H.I. Freedman studies cancer, which originates from the self-cells of the patient, but which then become hostile by mutations. There is much in common between cancer cells and pathogens originated from outside of the host body. Freedman discusses the optimal chemotherapy, considering the cost and benefit of chemotherapy.

This collection of papers gives an overview of theoretical studies of infectious disease dynamics and evolution, and hopefully will serve as a source in future studies of different aspects of infectious disease dynamics. Here, the key words are time delay, spatial dynamics, and evolution.

Toward the end of this introductory chapter, we would like to note one limitation - all of the papers in this volume discuss deterministic models, which are accurate when the population size is very large. Since the number of microparasites, such as bacteria, or viruses, or cancer cells, is often very large, the neglect of stochasticity due to the finiteness of individuals seems to be acceptable. However, when we consider the speed of the appearance of novel mutants, we do need stochastic models, because mutants always start from a small number. According to studies on the timing of cancer initiation, which starts from rare mutations followed by population growth of cancer cells, the predictions of deterministic models differ by several orders of magnitude from those of stochastic models and direct computer simulations.

\section{References}

1. Anderson, R. M. and R. M. May (1991), Infectious diseases of humans. Oxford University Press, Oxford UK.

2. Kermack, W. O. and A. G. McKendrick (1927), A contribution to the mathematical theory of epidemics. Proc. Roy. Soc. A 115, 700-721.

3. Shigesada, N. and K. Kawasaki (1997), Biological Invasions: Theory and Practice. Oxford University Press, Oxford. 